ANNALES

POLONICI MATHEMATICI

$95.1(2009)$

\title{
Uniqueness theorems and normal families of entire functions and their derivatives
}

by Feng Lü (Jinan), Junfeng Xu (Jiangmen) and Hongxun Yi (Jinan)

Abstract. We use the theory of normal families to obtain some uniqueness theorems for entire functions, which improve and generalize the related results of Rubel and Yang, and $\mathrm{Li}$ and Yi. Some examples are provided to show the sharpness of our results.

1. Introduction and main results. Let $f(z)$ and $g(z)$ be two nonconstant meromorphic functions in the complex plane $\mathbb{C}$ and let $a$ be a complex number. If $g(z)=a$ whenever $f(z)=a$, we write $f(z)=a \Rightarrow g(z)=a$. If $f(z)=a \Rightarrow g(z)=a$ and $g(z)=a \Rightarrow f(z)=a$, we write $f(z)=a \Leftrightarrow$ $g(z)=a$ and say that $f$ and $g$ share the value a IM (ignoring multiplicity). If $f-a$ and $g-a$ have the same zeros with the same multiplicities, we write $f(z)=a \rightleftharpoons g(z)=a$ and say that $f$ and $g$ share the value $a C M$ (counting multiplicity) (see [13]). It is assumed that the reader is familiar with the standard symbols and fundamental results of Nevanlinna theory, as found in $[3,13]$.

In 1977, Rubel and Yang [10] proved the well-known theorem.

Theorem A. Let $a$ and $b$ be complex numbers such that $b \neq a$, and let $f(z)$ be a nonconstant entire function. If $f(z)$ and $f^{\prime}(z)$ share the values a and $b C M$, then $f=f^{\prime}$.

This result has undergone various extensions and improvements (see [13]). Mues and Steinmetz [7] proved

THEOREM B. Let $a$ and $b$ be complex numbers such that $b \neq a$, and let $f(z)$ be a non-constant entire function. If $f(z)=a \Leftrightarrow f^{\prime}(z)=a$ and $f(z)=b \Leftrightarrow f^{\prime}(z)=b$, then $f \equiv f^{\prime}$.

Recently, Li and Yi [5] proved the following related results.

2000 Mathematics Subject Classification: 30D35, 30D45.

Key words and phrases: entire functions, Nevanlinna theory, uniqueness, normal family. 
Theorem C. Let $a$ and $b$ be complex numbers such that $b \neq a, 0$, and let $f(z)$ be a nonconstant entire function. If $f(z)=a \rightleftharpoons f^{\prime}(z)=a$ and $f^{\prime}(z)=b \Rightarrow f(z)=b$, then $f \equiv f^{\prime}$.

TheOREM D. Let $a$ and $b$ be complex numbers such that $b \neq a, 0$, and let $f(z)$ be a nonconstant entire function. If $f(z)=a \Rightarrow f^{\prime}(z)=a$ and $f(z)=b \rightleftharpoons f^{\prime}(z)=b$, then one of the following cases must occur:

(1) $f \equiv f^{\prime}$

(2) $f=C e^{b z /(b-a)}+a$, where $C$ is a nonzero constant.

It is natural to ask whether the conclusion of Theorem $\mathrm{C}$ remains valid if the hypothesis that $f$ and $f^{\prime}$ share $a$ CM is replaced by $f$ and $f^{\prime}$ sharing $a$ IM. In the present paper, we answer this question by proving the following result.

THEOREM 1.1. Let $a$ and $b$ be two nonzero distinct complex numbers, and let $f(z)$ be a nonconstant entire function. If $f(z)=a \Leftrightarrow f^{\prime}(z)=a$ and $f^{\prime}(z)=b \Rightarrow f(z)=b$, then $f \equiv f^{\prime}$.

The following two examples show that the conditions $a \neq 0$ and $b \neq 0$ cannot be omitted in Theorem 1.1.

ExAmple 1. Let $f=(b / 4) z^{2}$. Then

$$
f(z)=0 \Leftrightarrow f^{\prime}(z)=0 \text { and } f^{\prime}(z)=b \Rightarrow f(z)=b,
$$

but $f \not \equiv f^{\prime}$.

ExAmple 2. Let $f=A e^{(a / A) z}+a-A$, where $A$ is a nonzero constant. Then

$$
f(z)=a \Leftrightarrow f^{\prime}(z)=a \text { and } f^{\prime}(z) \neq 0,
$$

but $f \not \equiv f^{\prime}$.

Similarly, we can ask whether Theorem D still holds when the hypothesis that $f(z)=b \rightleftharpoons f^{\prime}(z)=b$ is replaced by $f(z)=b \Rightarrow f^{\prime}(z)=b$. The answer is negative in general, as shown by the following example.

ExAmple 3. Let $f=e^{z / 2}+2 e^{z / 4}+1$. It is easy to see that

$$
f=0 \Rightarrow f^{\prime}=0 \text { and } f=1 \Rightarrow f^{\prime}=1,
$$

but $f$ does not satisfy any case of Theorem $\mathrm{D}$.

However, we shall prove the following theorem:

THEOREM 1.2. Let $a$ and $b$ be complex numbers such that $b \neq a, 0$, and let $f(z)$ be a nonconstant entire function. If $f(z)=a \Rightarrow f^{\prime}(z)=a$ and $f(z)=b \Rightarrow f^{\prime}(z)=b$, then one of the following cases must occur:

(a) $f \equiv f^{\prime}$

(b) $f=A e^{b z /(b-a)}+a$ with $a \neq 0$, 
(c) $f=A e^{a z /(a-b)}+b$ with $a \neq 0$,

(d) $f=b\left\{\frac{1}{4} A^{2} e^{z / 2}+A e^{z / 4}+1\right\}$ with $a=0$,

where $A$ is a nonzero constant.

From the proof of Theorem 1.2, we can easily get the following corollary.

Corollary 1.1. Let $b$ be a nonzero number, and let $f(z)$ be a nonconstant entire function. If $f(z)=0 \Rightarrow f^{\prime}(z)=0$ and $f(z)=b \Rightarrow f^{\prime}(z)=b$, then one of the following cases must occur:

(a) $f \equiv f^{\prime}$,

(b) $f=b\left\{\frac{1}{4} A^{2} e^{z / 2}+A e^{z / 4}+1\right\}$, where $A$ is a nonzero constant.

REMARK 1.1. Obviously, Theorems 1.1 and 1.2 partially improve the former theorems.

It does not seem that the above theorems can be proved by using the methods in $[5,7]$. In order to prove our theorems, we need the following result relating to normal families, which is interesting in its own right.

THEOREM 1.3. Let $\mathcal{F}$ be a family of functions holomorphic on a domain $D$, and let $a$ and $b$ be distinct complex numbers. If for all $f \in \mathcal{F}$, $f(z)=a \Rightarrow f^{\prime}(z)=a$ and $f(z)=b \Rightarrow f^{\prime}(z)=b$, then $\mathcal{F}$ is normal in $D$.

In 1999, Xu [12] proved the following theorem.

THEOREM E. Let $\mathcal{F}$ be a family of functions holomorphic on a domain D, and let $a$ and $b$ be distinct complex numbers. If for all $f \in \mathcal{F}, f(z)=a \Leftrightarrow$ $f^{\prime}(z)=a$ and $f(z)=b \Leftrightarrow f^{\prime}(z)=b$, then $\mathcal{F}$ is normal in $D$.

Obviously, Theorem 1.3 improves Theorem E.

\section{Some lemmas}

LEMma $2.1([9])$. Let $\mathcal{F}$ be a family of functions holomorphic on the unit disc, and suppose that there exists $A \geq 1$ such that $\left|f^{\prime}(z)\right| \leq A$ whenever $f \in \mathcal{F}$ and $f(z)=0$. If $\mathcal{F}$ is not normal, then there exist, for each $0 \leq \alpha \leq 1$,

(a) a number $0<r<1$,

(b) points $z_{n}$ with $\left|z_{n}\right|<1$,

(c) functions $f_{n} \in \mathcal{F}$,

(d) positive numbers $a_{n} \rightarrow 0$ such that

$$
a_{n}^{-\alpha} f_{n}\left(z_{n}+a_{n} \xi\right)=g_{n}(\xi) \rightarrow g(\xi)
$$

locally uniformly, where $g$ is a nonconstant holomorphic function on $\mathbb{C}$ with order at most 1 such that $g^{\sharp}(\xi) \leq g^{\sharp}(0)=A+1$, where

$$
g^{\sharp}(\xi)=\frac{\left|g^{\prime}(\xi)\right|}{1+|g(\xi)|^{2}}
$$

is the spherical derivative. 
LEMma $2.2([5])$. Let $\mathcal{F}$ be a family of functions holomorphic on a domain $D$, and let $a$ and $b$ be distinct complex numbers such that $b \neq 0$. If for all $f \in \mathcal{F}, f(z)=a \Rightarrow f^{\prime}(z)=a$ and $f^{\prime}(z)=b \Rightarrow f(z)=b$, then $\mathcal{F}$ is normal in $D$.

Lemma $2.3([2,6])$. Let $f$ be an entire function and $M$ be a positive number. If $f^{\sharp}(z) \leq M$ for any $z \in \mathbb{C}$, then $f$ is of exponential type.

LEMMA 2.4 ([11]). Let $\zeta$ be a family of meromorphic functions in a domain $D$. Then $\zeta$ is normal in $D$ if and only if the spherical derivatives of functions $f \in \zeta$ are uniformly bounded on compact subsets of $D$.

Lemma 2.5 ([8], [4, Theorem 4.1]). Let $f$ be an entire function of order at most 1 , and $k$ be a positive integer. Then

$$
m\left(r, f^{(k)} / f\right)=o(\log r) \quad \text { as } r \rightarrow \infty .
$$

3. Proof of Theorem 1.3. Since normality is a local property, we may assume that the domain $D$ is the unit disc. Suppose, to the contrary, that $\mathcal{F}$ is not normal in $D$. Since $a \neq b$, we can assume $b \neq 0$. Set $\mathcal{F}_{1}=\{f-a$ : $f \in \mathcal{F}\}$. Then $\mathcal{F}_{1}$ is not normal in $D$. By assumption, for any function $h(z)=f(z)-a$, we have $\left|h^{\prime}(z)\right| \leq|a|+1$ whenever $h(z)=0$. By Lemma 2.1 (with $\alpha=0$ and $A=|a|+1$ ), there exist $f_{n}-a \in \mathcal{F}_{1}, z_{n} \in D$, and $a_{n} \rightarrow 0$ as $n \rightarrow \infty$ such that

$$
g_{n}(\xi)=f_{n}\left(z_{n}+a_{n} \xi\right)-a \rightarrow g(\xi)
$$

locally uniformly, $g$ is a nonconstant entire function with order $\varrho(g) \leq 1$, and

$$
g^{\sharp}(\xi) \leq g^{\sharp}(0)=|a|+2 .
$$

Suppose that $g\left(\eta_{0}\right)=0$. Hurwitz's theorem implies the existence of a sequence $\eta_{n} \rightarrow \eta_{0}$ with

$$
g_{n}\left(\eta_{n}\right)=f_{n}\left(z_{n}+a_{n} \eta_{n}\right)-a=0 .
$$

Since $f(z)=a \Rightarrow f^{\prime}(z)=a$, we have $f_{n}^{\prime}\left(z_{n}+a_{n} \eta_{n}\right)=a$. Then

$$
g^{\prime}\left(\eta_{0}\right)=\lim _{n \rightarrow \infty} g_{n}^{\prime}\left(\eta_{n}\right)=\lim _{n \rightarrow \infty} a_{n} f_{n}^{\prime}\left(z_{n}+a_{n} \eta_{n}\right)=0,
$$

and hence the zeros of $g(\xi)$ are of multiplicity at least 2. Similarly, we find that the zeros of $g(\xi)-(b-a)$ are of multiplicity at least 2 .

Next, we shall prove that $g(\xi) \neq b-a$. Suppose $\xi_{0}$ is a zero of $g(\xi)-(b-a)$ with multiplicity $m(\geq 2)$. Then $g^{(m)}\left(\xi_{0}\right) \neq 0$. Thus there exists a positive number $\delta$ such that

$$
g(\xi) \neq 0, \quad g^{\prime}(\xi) \neq 0, \quad g^{(m)}(\xi) \neq 0
$$

on $D_{\delta}^{o}=\left\{z: 0<\left|\xi-\xi_{0}\right|<\delta\right\}$.

Noting that $g(\xi) \not \equiv b-a$, by the Rouché theorem, there exist $\xi_{n, j}$ $(j=1, \ldots, m)$ in $D_{\delta / 2}=\left\{\xi:\left|\xi-\xi_{0}\right|<\delta / 2\right\}$ such that $g_{n}\left(\xi_{n, j}\right)=b-a$. 
Since

$$
g_{n}^{\prime}\left(\xi_{n, j}\right)=a_{n} f_{n}^{\prime}\left(z_{n}+a_{n} \xi_{n, j}\right)=a_{n} b \neq 0 \quad(j=1, \ldots, m),
$$

each $\xi_{n, j}$ is a simple zero of $g_{n}(\xi)-(b-a)$, that is, $\xi_{n, j} \neq \xi_{n, i}(1 \leq i \neq j \leq m)$.

On the other hand,

$$
\lim _{n \rightarrow \infty} g_{n}^{\prime}\left(\xi_{n, j}\right)=\lim _{n \rightarrow \infty} a_{n} b=0 .
$$

From (3.3), we have

$$
\lim _{n \rightarrow \infty} \xi_{n, j}=\xi_{0} \quad(j=1, \ldots, m) .
$$

By (3.3), since $g_{n}^{\prime}(\xi)-a_{n} b$ has $m$ zeros $\xi_{n, j}(j=1, \ldots, m)$ in $D_{\delta / 2}$, it follows that $\xi_{0}$ is a zero of $g^{\prime}(\xi)$ with multiplicity $m$, and thus $g^{(m)}\left(\xi_{0}\right)=0$. This is a contradiction. Hence $g(\xi) \neq b-a$.

By the Nevanlinna second fundamental theorem, we arrive at a contradiction. This completes the proof of Theorem 1.3.

4. Proof of Theorem 1.1. First, we prove that $f$ is of exponential type. Set $\mathcal{F}=\{f(z+w): w \in \mathbb{C}\}$. Then $\mathcal{F}$ is a family of holomorphic functions on the unit disc $\triangle$. By assumption, for any function $h(z)=f(z+w)$, we have $h(z)=a \Rightarrow h^{\prime}(z)=a$ and $h^{\prime}(z)=b \Rightarrow h(z)=b$ for all $z \in \triangle$. Hence by Lemma $2.2, \mathcal{F}$ is normal in $\triangle$. Thus by Lemma 2.4 , there exists $M>0$ satisfying $f^{\sharp}(z) \leq M$ for all $z \in \mathbb{C}$. From Lemma 2.3, we deduce that $f$ is of exponential type. Since $f(z)=a \Leftrightarrow f^{\prime}(z)=a$, it follows that $f$ is a transcendental entire function.

Set

$$
g=\frac{\left(f^{\prime}-f\right) f^{\prime \prime}}{(f-a)\left(f^{\prime}-b\right)} .
$$

From the assumption that $f=a \Leftrightarrow f^{\prime}=a$ and $f^{\prime}=b \Rightarrow f=b$, we derive that $g$ is an entire function. By (4.1), we have

$$
\begin{aligned}
g & =\frac{f^{\prime}}{f-a} \frac{f^{\prime \prime}}{f^{\prime}-b}-\frac{(f-a+a) f^{\prime \prime}}{(f-a)\left(f^{\prime}-b\right)} \\
& =\frac{f^{\prime}}{f-a} \frac{f^{\prime \prime}}{f^{\prime}-b}-\frac{f^{\prime \prime}}{f^{\prime}-b}-\frac{f^{\prime}}{f-a} \frac{a f^{\prime \prime}}{\left(f^{\prime}-b\right) f^{\prime}} .
\end{aligned}
$$

Thus, by Lemma 2.5,

$$
\begin{aligned}
T(r, g)=m(r, g) \leq & m\left(r, \frac{f^{\prime}}{f-a} \frac{f^{\prime \prime}}{f^{\prime}-b}\right)+m\left(r, \frac{f^{\prime \prime}}{f^{\prime}-b}\right) \\
& +m\left(r, \frac{f^{\prime}}{f-a} \frac{a f^{\prime \prime}}{\left(f^{\prime}-b\right) f^{\prime}}\right)=o(\log r),
\end{aligned}
$$

which implies that $g$ is a constant. We can write $g=c$. 
Suppose that $c \neq 0$. By (4.1) we find that $f^{\prime}-a$ has only simple zeros, hence $f=a \rightleftharpoons f^{\prime}=a$. From Theorem C we obtain $f \equiv f^{\prime}$, thus $g=c=0$, a contradiction.

Suppose now that $c=0$. Then $f \equiv f^{\prime}$ or $f^{\prime \prime} \equiv 0$. If $f^{\prime \prime} \equiv 0$, then $f$ is a polynomial, which is a contradiction. Thus $f \equiv f^{\prime}$.

5. Proof of Theorem 1.2. Set $\mathcal{F}=\{f(z+w): w \in \mathbb{C}\}$. Then $\mathcal{F}$ is a family of holomorphic functions on the unit disc $\triangle$. By assumption, for each function $l(z)=f(z+w)$, we have $l(z)=a \Rightarrow l^{\prime}(z)=a$ and $l(z)=b \Rightarrow l^{\prime}(z)=b$ for all $z \in \triangle$. Hence by Theorem $1.3, \mathcal{F}$ is normal in $\triangle$. Thus by Lemma 2.4, there exists $M>0$ satisfying $f^{\sharp}(z) \leq M$ for all $z \in \mathbb{C}$. By Lemma 2.3, $f$ is of exponential type.

Suppose that $f$ is a polynomial. From $f=b \Rightarrow f^{\prime}=b$, we deduce that $f$ is a linear polynomial and $f=b z+d$, where $d$ is a constant. As $f=a \Rightarrow f^{\prime}=a$, we obtain a contradiction. So $f$ is a transcendental entire function.

In the following, we consider two cases:

CaSe 1: $a b \neq 0$. Set

$$
\mu=\frac{f^{\prime}\left(f-f^{\prime}\right)}{(f-a)(f-b)} .
$$

Then $\mu$ is an entire function and

$$
\begin{aligned}
T(r, \mu) & =m(r, \mu) \\
& \leq m\left(r, \frac{f^{\prime} f}{(f-a)(f-b)}\right)+m\left(r, \frac{f^{\prime} f^{\prime}}{(f-a)(f-b)}\right)+O(1) \\
& =m\left(r, \frac{1}{b-a}\left(\frac{b f^{\prime}}{f-b}-\frac{a f^{\prime}}{f-a}\right)\right)+m\left(r, \frac{f^{\prime} f^{\prime}}{(f-a)(f-b)}\right)+O(1) .
\end{aligned}
$$

By Lemma 2.5, we see that $T(r, \mu)=o(\log r)$, thus $\mu$ is a constant.

If $\mu=0$, then $f \equiv f^{\prime}$.

Suppose $\mu \neq 0$. Thus, $f^{\prime} \neq 0$. Set

$$
f^{\prime}(z)=A e^{\lambda z},
$$

where $A$ and $\lambda$ are two nonzero constants. Then

$$
f=c+\frac{A}{\lambda} e^{\lambda z},
$$

where $c$ is a constant. Next we consider three subcases:

Subcase 1.1: $a$ is not a Picard value of $f$. Then $a \neq c$. Let $z_{0}$ be a zero of $f-a$. By (5.2) and (5.3), we have

$$
\lambda=\frac{a}{a-c} .
$$

Then $f=c+A e^{a z /(a-c)}$. 
Suppose $b$ is a Picard value of $f$. Then $c=b$. Thus $f=b+A e^{a z /(a-b)}$.

Now assume that $b$ is not a Picard value of $f$. Let $b_{0}$ be a zero of $f^{\prime}-b$. By (5.2) and (5.3), we have $c=0$ and $f=A e^{z}$, thus $f \equiv f^{\prime}$.

Subcase 1.2: $b$ is not a Picard value of $f$. Similarly to Case 1 , we deduce $f=a+A e^{b z /(b-a)}$.

Subcase 1.3: $a$ and $b$ are two Picard values of $f$. Then by Nevanlinna's second fundamental theorem, we arrive at a contradiction.

CASE 2: $a b=0$. Then $a=0$. Let $h=f / b$. Then $h=0 \Rightarrow h^{\prime}=0$, $h=1 \Rightarrow h^{\prime}=1$ and $\{h(z+\omega): \omega \in \mathbb{C}\}$ is normal in $\Delta$. Then $h$ is of exponential type. Set

$$
g=\frac{h^{\prime}\left(h^{\prime}-h\right)}{h(h-1)} .
$$

We see that $g$ is a entire function. As above, we have

$$
T(r, g)=m(r, g)=o(\log r),
$$

thus $g$ is a constant.

If $g=0$, then $h \equiv h^{\prime}$ and $f \equiv f^{\prime}$.

Now, we assume that $g \neq 0$. By (5.5) we have

$$
h^{\prime}\left(h^{\prime}-h\right)=g h(h-1) .
$$

Taking the derivative, we obtain

$$
h^{\prime \prime}\left(h^{\prime}-h\right)+h^{\prime}\left(h^{\prime \prime}-h^{\prime}\right)=g\left[h^{\prime}(h-1)+h h^{\prime}\right]
$$

and

$$
h^{\prime \prime \prime}\left(h^{\prime}-h\right)+2 h^{\prime \prime}\left(h^{\prime \prime}-h^{\prime}\right)+h^{\prime}\left(h^{\prime \prime \prime}-h^{\prime \prime}\right)=g\left[h^{\prime \prime}(h-1)+2 h^{2}+h h^{\prime \prime}\right] .
$$

If $h \neq 1$, we set $h=A_{1} e^{\lambda_{1} z}+1$, where $A_{1}$ and $\lambda_{1}$ are nonzero constants. From $h=0 \Rightarrow h^{\prime}=0$, we get a contradiction. Thus $h-1$ has zeros.

Let $c_{0}$ be a zero of $h-1$. By (5.6), we get $h^{\prime \prime}\left(c_{0}\right)=g+1$, which yields $h=1 \Rightarrow h^{\prime \prime}=g+1$.

Put

$$
\phi=\frac{h^{\prime \prime}-(1+g) h^{\prime}}{h-1}
$$

this is an entire function. Similarly to the above, we find that $\phi$ is a constant. Taking the derivative, we have

$$
\phi h^{\prime}=h^{\prime \prime \prime}-(1+g) h^{\prime \prime} .
$$

Substitute $c_{0}$ into (5.7) and (5.9) to obtain

$$
\phi=-2 g^{2} .
$$


If 0 is a Picard value of $h$, we set $h=A_{2} e^{\lambda_{2} z}$, where $A_{2}, \lambda_{2}$ are nonzero constants. From $h=1 \Rightarrow h^{\prime}=1$, we derive that $\lambda_{2}=1$. Thus $h \equiv h^{\prime}$ and $g \equiv 0$, a contradiction. Hence 0 is not a Picard value of $h$.

Let $a_{0}$ be a zero of $h$. Then from (5.7), we deduce

$$
2 h^{\prime \prime}\left(a_{0}\right)^{2}=-g h^{\prime \prime}\left(a_{0}\right) \text {. }
$$

If $h^{\prime \prime}\left(a_{0}\right)=0$, then $a_{0}$ is a multiple zero of $f^{\prime}-f$. By (5.5), we find $g=0$, a contradiction.

Hence, $h^{\prime \prime}\left(a_{0}\right) \neq 0$ and $2 h^{\prime \prime}\left(a_{0}\right)=-g$. Put $a_{0}$ into (5.8) to find $h^{\prime \prime}\left(a_{0}\right)=$ $-\phi$. This yields $g=2 \phi$.

Thus, we get $g=-1 / 4$ and $\phi=-1 / 8$. By (5.8), we have

$$
h^{\prime \prime}-\frac{3}{4} h^{\prime}+\frac{1}{8}(h-1)=0 .
$$

Solving the equation gives

$$
h=C_{1} e^{z / 2}+C_{2} e^{z / 4}+1 .
$$

From $h=0 \Rightarrow h^{\prime}=0, h=1 \Rightarrow h^{\prime}=1$, we get $4 C_{1}=C_{2}^{2}$. Hence

$$
f=b\left\{\frac{1}{4} C_{2}^{2} e^{z / 2}+C_{2} e^{z / 4}+1\right\} .
$$

REMARK 5.1. For further study, we ask some questions.

Question 1. What will happen if $b=0$ in Theorem 1.1?

Question 2. What will happen if the hypothesis $f(z)=a \Leftrightarrow f^{\prime}(z)=a$ is replaced by $f(z)=a \Rightarrow f^{\prime}(z)=a$ in Theorem 1.1?

Unfortunately, we cannot answer the questions with the above methods.

Acknowledgements. We are grateful to the referee for pointing out Lemma 2.5 which let us simplify our proofs.

This project was supported by the NSFC (No. 10771121) and the NSFCRFBR.

\section{References}

[1] C. A. Berenstein and R. Gay, Complex Variables, Springer, New York, 1991.

[2] J. Clunie and W. K. Hayman, The spherical derivative of integral and meromorphic functions, Comment. Math. Helv. 40 (1966), 117-148.

[3] W. Hayman, Meromorphic Functions, Clarendon Press, Oxford, 1964.

[4] J. Heittokangas, R. Korhonen, and J. Rättyä, Generalized logarithmic derivative estimates of Gol'dberg-Grinshtein type, Bull. London Math. Soc. 36 (2004), 105114 .

[5] J. T. Li and H. X. Yi, Normal families and uniqueness of entire functions and their derivatives, Arch. Math. (Basel) 87 (2006), 52-59.

[6] D. Minda, Yosida functions, in: Lectures on Complex Analysis (Xian, 1987), C. T. Chuang (ed.), World Sci., Singapore, 1988, 197-213.

[7] E. Mues und N. Steinmetz, Meromorphe Funktionen, die mit ihrer Ableitung Werte teilen, Manuscripta Math. 29 (1979), 195-206. 
[8] V. Ngoan and I. V. Ostrovskii, The logarithmic derivative of a meromorphic function, Akad. Nauk. Armyan. SSR Dokl. 41 (1965), 272-277 (in Russian).

[9] X. C. Pang and L. Zalcman, Normal families and shared values, Bull. London Math. Soc. 32 (2000), 325-331.

[10] L. A. Rubel and C. C. Yang, Values shared by an entire function and its derivative, in: Complex Analysis, Lecture Notes in Math. 599, Springer, Berlin, 1976, 101-103.

[11] J. Schiff, Normal Families, Springer, 1993.

[12] Y. Xu, Normality criteria concerning sharing values, Indian J. Pure Appl. Math. 30 (1999), 287-293.

[13] H. X. Yi and C. C. Yang, Uniqueness Theory of Meromorphic Functions, Science Press, Beijing, 1995.

School of Mathematics

Shandong University

Jinan, Shandong, 250100, P.R. China

E-mail: lvfeng18@gmail.com

hxyi@sdu.edu.cn
Department of Mathematics

Wuyi University

Jiangmen, Guangdong, 529020, P.R. China

E-mail: xujunf@gmail.com

Received 27.5.2008

and in final form 20.9.2008 